\title{
ON THE SPECTRUM OF ALGEBRAIC $K$-THEORY
}

\author{
BY S. M. GERSTEN
}

Communicated by H. Bass, October 4, 1971

\begin{abstract}
The groups $K_{i}(A)$ of Bass for $i<0$ are identified as homotopy groups of the spectrum of algebraic $K$-theory. The spectrum itself is identified. Applications to Laurent polynomials and to $K$-theory exact sequences are given.
\end{abstract}

Quillen has recently proposed a $K$-theory for unital rings [12], [13]. He associates to a ring $A$ a space $B G 1(A)^{+}$whose homology is that of the group $G 1(A)$ and whose homotopy groups $\pi_{i} B G 1(A)^{+}$he defines as $K_{i}(A)$, $i \geqq 1$. The space $B G 1(A)^{+}$is known to be an $H$-space, and indeed an infinite loop space.

Hence one is motivated to define $K_{i}(A)$, for $i \in Z$, as $\pi_{i}(E(A))$ where $E(A)$ is the associated $\Omega$-spectrum. This note describes $E(A)$ and identifies the groups $K_{i}(A), i<0$. In fact, we show that the groups $K_{i}(A)$ are exactly the groups $L^{-i} K_{0}(A)$ discussed in Bass' book [3, p. 664] for $i<0$.

Recall from the work of Karoubi and Villamayor [10] the cone $C A$ and suspension $S A$ of a ring $A$. An infinite matrix is called permutant if it is an infinite permutation matrix times a diagonal matrix of finite type. The diagonal matrix is of finite type if its diagonal entries are chosen from a finite subset of the ring. The ring $C A$ is the ring generated by permutant matrices. The cone $C A$ contains the two-sided ideal $\tilde{A}=\bigcup_{n} M_{n}(A)$ and the quotient ring is called the suspension of $A$. We can now state our main result.

THEOREM A. The space $\Omega\left(B G 1(S A)^{+}\right)$has the homotopy type of $K_{0}(A) \times B G 1(A)^{+}$

Corollary. For all $i \in Z$ we have $K_{i}(A)=K_{i+1}(S A)$.

Since Karoubi [9] has already identified $K_{0}\left(S^{i} A\right)$ with Bass' groups $K_{-i}(A)$, the Corollary above completes the identification of Bass' groups with the negative homotopy of the spectrum $E(A)$.

In proving Theorem A we must first analyze the cone construction.

THEOREM B. The space $B G 1(C A)^{+}$is contractible.

This result generalizes work of Karoubi and Villamayor [11] who show that $K_{i}(C A)=0$ for $i \leqq 2$. To prove Theorem $\mathrm{B}$ we observe that it suffices

AMS 1970 subject classifications. Primary 18F25, 55B15, 16A54, 13D15, 55F50, 18G30, 55B20, 55D35.

Key words and phrases. Algebraic $K$-theory, suspension and cone of a ring, $\Omega$-spectrum, flasque category, locally free simplicial monoid, group completion functor, Laurent polynomials. 
to show that $\tilde{H}_{*}\left(B G 1(C A)^{+}\right)=0$. This latter result is a consequence of a theorem of Karoubi [7] that the category $\mathscr{P}(C A)$ is "flasque," together with a recent result of Barratt and Priddy [2] which implies

THEOREM C. Suppose that $M$ is a locally free simplicial monoid (i.e. $M_{n}$ is a free monoid for all $n$ ). Let $\hat{M}$ denote the group completion of $M$. If $H_{*}(M, Z)$ is (graded) commutative, then $\pi_{0}(\hat{M})=\left(\pi_{0}(\hat{M})\right)$ and $H_{*}(\hat{M}, Z)$ $=H_{*}(M, X) \otimes_{Z \pi_{0}(M)} Z\left[\pi_{0}(\hat{M})\right]$.

D. W. Anderson informed me that Quillen has also given a proof of Theorem C.

In order to apply Theorem $\mathrm{C}$, it is necessary to use the description of $B G 1(C A)^{+}$given by D. W. Anderson [1] as the group completion of the morphism complex of the "blown-up" permutative category $\mathscr{P}(C A)$.

Let $I$ be the image of $G 1(C A)$ in $G 1(S A)$. Since $K_{1}(C A)=0$ it follows that $I=E(S A)$, the elementary group of matrices. We have two short exact sequences of groups

and

$$
G 1(\tilde{A}) \rightarrow G 1(C A) \rightarrow E(S A),
$$

$$
E(S A) \rightarrow G 1(S A) \rightarrow K_{1}(S A) .
$$

Recall $Z_{\infty}$, the integral completion functor of Bousfield and Kan [4], [5]. We remark that $Z_{\infty} B G 1(A) \simeq B G 1(A)^{+}$, as was established in [6]. The following theorem was communicated to us directly by Bousfield.

Theorem D (A. K. Bousfield). Let $F \rightarrow E \rightrightarrows \rightarrow B$ be a fibration of connected spaces and suppose (1) $Z_{\infty} E$ and $Z_{\infty} B$ are nilpotent and (2) $\pi_{1}(B)$ acts nilpotently on each $H_{n}(F)$. Then the inclusion of $Z_{\infty} F$ in the fibre of the map $Z_{\infty} \pi$ is a homotopy equivalence, and moreover $Z_{\infty} F$ is nilpotent.

One checks the hypotheses of Theorem $D$ for the fibration

$$
B E(S A) \rightarrow B G 1(S A) \rightarrow B K_{1}(S A) .
$$

The action of $K_{1}(S A)$ on $H_{*}(B E(S A))$ is the limit of inner automorphisms, and hence is trivial. We deduce that we have a fibration

$$
Z_{\infty} B E(S A) \rightarrow Z_{\infty} B G 1(S A) \rightarrow B K_{1}(S A)
$$

with $Z_{\infty} B E(S A)$ nilpotent. In fact, $Z_{\infty} B E(S A)$ is easily seen to be the universal cover of $B G 1(S A)^{+}$.

Consider now the commutative diagram whose rows are fibrations

$$
\begin{array}{ccc}
F_{0} & \rightarrow Z_{\infty} B G 1(C A) \rightarrow Z_{\infty} B E(S A) \\
\downarrow & \downarrow= & \downarrow \\
F & \rightarrow Z_{\infty} B G 1(C A) \rightarrow Z_{\infty} B G 1(S A) .
\end{array}
$$


After examining the exact homotopy sequences, it follows that $F_{0}$ is a connected component of $F$. One considers now the fibration

$$
B G 1(\tilde{A}) \rightarrow B G 1(C A) \rightarrow B E(S A) .
$$

Again one checks that the hypotheses of Theorem $D$ are satisfied. Here the action of $E(S A)$ on $H_{*}(B G 1(\tilde{A}))$ is trivial. The idea for this observation is already contained in [9] in the proof of Lemma 5.9. It follows that $Z_{\infty} B G 1(\tilde{A}) \simeq F_{0}$. Next we establish

LEMma. $Z_{\infty} B G 1(\tilde{A}) \simeq Z_{\infty} B G 1(A)$.

This Lemma generalizes [11, Proposition 7.4]. After an application of Theorem B, the proof of Theorem A is quickly completed.

Following Karoubi [9] we observe that the Corollary of Theorem A has application to the study of Laurent polynomials.

TheOREM E. For any unital ring $A$ and $n \in Z$ we have

$$
K_{n}\left(A\left[t, t^{-1}\right]\right)=K_{n}(A) \oplus K_{n-1}(A) \oplus ? .
$$

One considers the pairing

$$
K_{n-1}(A) \otimes K_{1}\left(Z\left[t, t^{-1}\right]\right) \stackrel{\cup}{\rightarrow} K_{n}\left(A\left[t, t^{-1}\right]\right)
$$

which induces a map $K_{n-1}(A) \rightarrow K_{n}\left(A\left[t, t^{-1}\right]\right)$ by $x \mapsto x \cup[t]$. Karoubi's idea to exhibit an inverse to this map is as follows. He defines a homomorphism $A\left[t, t^{-1}\right] \rightarrow S A$ by sending $\sum a_{i} t^{i}$ to the coset of the matrix in $C A$ :

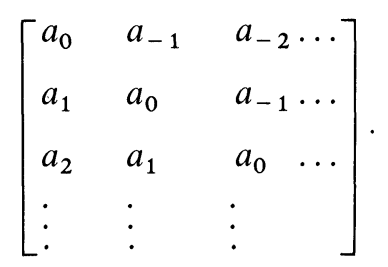

This induces the map $K_{n}\left(A\left[t, t^{-1}\right]\right) \rightarrow K_{n}(S A)$. By the Corollary to Theorem A, $K_{n}(S A)=K_{n-1}(A)$ and one checks that the resulting map is a left inverse to the cup product map.

We also have results giving a homotopy theoretic interpretation to the $K$-theoretic exact sequences of a surjection. These $K$-theory sequences may be deduced from

THEOREM F. Let $q$ be a two-sided ideal in the ring $A$. Let $F$ be the homotopy theoretic fibre of the induced map $B G 1(A)^{+} \rightarrow B G 1(A / q)^{+}$. Then there is a canonical isomorphism $\pi_{1} F \cong K_{1}(A, q)$. 
I now know $K$-theoretic interpretations for the higher homotopy groups of the fibre $F$. It would be very interesting to interpret the corresponding fibre in the case of a localization $A \rightarrow A_{S}$.

\section{BIBLIOGRAPHY}

1. D. W. Anderson, Simplicial K-theory and generalized homology theories. I. Examples from $K$-theory (preprint).

2. M. Barratt and S. Priddy, On the homology of non connected monoids and their associated groups (to appear).

3. H. Bass, Algebraic K-theory, Benjamin, New York, 1968. MR 40 \# 2736.

4. A. K. Bousfield and D. M. Kan, Homotopy with respect to a ring, Proc. Sympos. Pure Math., vol. 22, Amer. Math. Soc., Providence, R.I., 1971.

5. - - The homotopy spectral sequence of a space with coefficients in a ring (to appear).

6. S. M. Gersten and D. L. Rector, A relation between two simplicial algebraic $K$-theories, Bull. Amer. Math. Soc. 77 (1971), 397-399.

7. M. Karoubi, Foncteurs dérivés et $K$-théorie, Séminaire sur la $K$-théorie, Lecture Notes in Math., vol. 136, Springer-Verlag, Berlin, 1970.

8. - La périodicité de Bott en K-théorie générale, C. R. Acad. Sci. Paris Sér. A-B 270 (1970), A1305-A1307. MR 41 \# 5462.

9., La périodicité de Bott en K-théorie générale, Ann. Sci. École Norm. Sup. 4 (1971), 63-95.

10. M. Karoubi and O. Villamayor, Foncteurs $K^{n}$ en algèbre et en topologie, C. R. Acad. Sci. Paris Sér. A-B 269 (1969), A416-A419. MR 40 \# 4944.

11. $-K$-théorie algébrique et $K$-théorie topologique (to appear).

12. D. Quillen, Cohomology of groups (to appear).

13. - The $K$-theory associated to a finite field. I (to appear).

14. G. Segal, Homotopy everything $H$-spaces (to appear).

Department of Mathematics, Rice University, Houston, Texas 77001 